\title{
A material and product audit method for determining the level of quality
}

\begin{abstract}
This paper introduces a method for evaluating material and product quality. The method is developed based on the normal distribution. For quantitative variable, the method divides the tolerance zones to 3 parts according to normal distribution \pm 3 and assigns different scores to tolerance region. For qualitative variables, the method take decision base on the fact that the measurement result can be occurred on two states as accept or reject. Therefore, a Level of Quality (LOQ) will be measured for the product quality based on the measurement of different samples of lot by quantitative and qualitative variables. The method is validated by a numerical example included 2 dimensions.
\end{abstract}

Keyword: Level of quality (LOQ); Normal distribution; Quality audit; Quality management system; Tolerance 